\title{
Non-equilibrium conductance of a three-terminal quantum dot in the Kondo regime: Perturbative Renormalization Group
}

\author{
N. Shah and A. Rosch \\ Institut für Theoretische Physik, Universität zu Köln, 50937 Köln, Germany.
}

\begin{abstract}
Motivated by recent experiments, we consider a single-electron transistor in the Kondo regime which is coupled to three leads in the presence of large bias voltages. Such a steady-state nonequilibrium system is to a large extent governed by a decoherence rate $\Gamma$ induced by the current through the dot. As the two-terminal conductance turns out to be rather insensitive to $\Gamma$, we study the conductance in a three-terminal device using perturbative renormalization group and calculate the characteristic splitting of the Kondo resonance. The interplay between potential biases and anisotropy in coupling to the three leads determines $\Gamma$ and the conditions for strong coupling.
\end{abstract}

With the advent of highly tunable strongly correlated systems it has not only become possible to systematically realize and investigate well-studied theoretical models but also to pose new theoretical questions of fundamental nature. Though non-equilibrium physics in itself has a long history, a lot remains to be unfolded in the context of strongly correlated quantum systems. Since the single-impurity Kondo model, describing a spin coupled to conduction electrons, is not only one of the best understood theoretical prototype but also the one that has state-of-the-art experimental realization in tunable nanosystems involving quantum dots [1, 2], single molecules [3, 4] and perhaps cold atoms in the near future [5], it is most natural and crucial to ask: what happens at the juncture of Kondo and non-equilibrium physics?

In equilibrium, the Kondo coupling grows from "weak" to "strong" on lowering the temperature and finally the spin is screened. An applied bias voltage $V$ influences the Kondo physics in at least two ways. First, it prohibits resonant spin-scattering processes among the leads by splitting the relevant Fermi energies by $V$. More interesting and less obvious [6, 7, 8, 9, 10] is the fate and role of intra-lead resonant spin-flip couplings. Will they grow on reducing the energy or will the problem remain weakly coupled for large voltages? At least for symmetric coupling, the Kondo model remains at weak coupling for $V \gg T_{K}$ ( $T_{K}$ being the equilibrium Kondo temperature), as the large current through the dot induces noise and decoheres the resonant spin-flip processes as shown in Refs. [8, 9, 10, 11]. The physics is then to a large extent controlled by the corresponding decoherence rate $\Gamma$. Therefore the question arises which experimental quantity can be used to investigate this second mechanism. Unfortunately, the usual two-terminal conductance is almost completely blind to this kind of physics as it it dominated by (non-resonant) inter-lead processes 12. However, a configuration where three leads are coupled to a quantum dot has much more flexibility and is indeed sensitive to $\Gamma$ as shown below.

Such systems have recently been realized by three groups [13, 14, 15]. Francheschi et al. 13] used a clever indirect realization in a dot connected to one lead and one mesoscopic wire driven out of equilibrium. The device of Leturcq et al., in contrast, is based on a small ring with three leads attached [14]. They managed to operate this device in the Kondo regime, where effectively a single spin is localized (or rather delocalized) on the ring. A magnetic field allows to fine-tune the wave function and therefore to adjust the exchange couplings to the leads. A weakly coupled third lead was proposed [16, 17, 18, 19] also as a way to experimentally see the splitting of the Kondo resonance by a bias voltage, which lies beyond the scope of a two-terminal measurement - this splitting has indeed been observed by these experiments.

In this letter, we carry out the non-equilibrium perturbative Renormalization Group (RG) analysis [1] of a three-terminal Kondoesque device and calculate the conductance in the presence of applied voltage biases. We model the system by the Hamiltonian

$H=\sum_{\alpha \mathbf{k} \sigma} \varepsilon_{\mathbf{k}} c_{\alpha \mathbf{k} \sigma}^{\dagger} c_{\alpha \mathbf{k} \sigma}+\frac{1}{3} \sum_{\alpha \alpha^{\prime} \mathbf{k} \mathbf{k}^{\prime} \sigma \sigma^{\prime}} J_{\alpha \alpha^{\prime}} S_{\lambda} c_{\alpha \mathbf{k} \sigma}^{\dagger} \sigma_{\sigma \sigma^{\prime}}^{\lambda} c_{\alpha^{\prime} \mathbf{k}^{\prime} \sigma^{\prime}}$

where $\alpha=1,2,3$ denote the three leads, each with a distribution function $f_{\alpha}(\omega)=1 /\left(1+\exp \beta\left(\omega-\mu_{\alpha}\right)\right)$ with the electrochemical potential $\mu_{\alpha}$ incorporating the applied voltages. The key point is that in non-equilibrium, all electrons in the energy windows corresponding to the applied voltages, contribute to the low energy properties and the effective renormalized exchange coupling of the electrons from the leads with the local "spin" depends on their energy. We derive the RG equations for these frequency-dependent coupling functions (denoted by $\left.g_{\alpha \alpha^{\prime}}(\omega)\right)$ to leading order in the small parameter $1 / \ln \left[\max \left(\left|\mu_{\alpha}-\mu_{\beta}\right|,\left|\omega-\mu_{\alpha}\right|\right) / T_{K}\right]$. The equations read

$$
\frac{\partial g_{\alpha \alpha^{\prime}}(\omega)}{\partial \ln D}=-\frac{2}{3} \sum_{\beta=1}^{3} g_{\alpha \beta}\left(\mu_{\beta}\right) g_{\beta \alpha^{\prime}}\left(\mu_{\beta}\right) \Theta\left(D-\left|\omega-\mu_{\beta}\right|\right)
$$

with the initial conditions at the bare cutoff $D=D_{0}$ given by $g_{\alpha \alpha^{\prime}}(\omega)=\rho J_{\alpha \alpha^{\prime}}$, where $\rho$ is the density of states. The meaning is transparent: At each value of the cut-off $D$, an electron of lead $\beta$ at $\mu_{\beta}$ contributes to the renormalization of $g_{\omega}(D)$ via a second order virtual process 
only for frequencies that lie within the range of the cutoff from $\mu_{\beta}$. By choosing $\omega=\mu_{\beta}$, the RG equations are first solved for all the constants $g_{\alpha \beta}\left(\mu_{\beta}\right)$ on the RHS to obtain their entire $D$-dependence, and then in the second step $g_{\alpha \alpha^{\prime}}(\omega)$ is obtained by using in the RHS the pre-evaluated constants at every $D$.

As we have emphasized, the crucial element in the nonequilibrium is the so-called decoherence rate $[$ ]

$$
\Gamma=\frac{4 \pi}{9 \hbar} \sum_{\alpha, \alpha^{\prime}} \int_{-\infty}^{\infty} d \omega g_{\alpha \alpha^{\prime}}^{2}(\omega) f_{\alpha}(\omega)\left(1-f_{\alpha^{\prime}}(\omega)\right)
$$

responsible for stopping the RG flow before the strong coupling regime given by the above $\mathrm{RG}$ equation is reached. Implementation of this effect in the RG procedure amounts to replacing the $\Theta$ function in Eq. (2) by $\Theta\left(D-\left|\widetilde{\omega-\mu_{\beta}}\right|\right)$ (with the short-hand $\widetilde{\omega} \equiv \sqrt{\omega^{2}+\Gamma^{2}}$ ) and evaluating the value of $\Gamma$ self-consistently.

When the Hamiltonian is assumed to be derived from an underlying Anderson model then the $J_{\alpha \alpha^{\prime}}$ are separable $\left(J_{\alpha \alpha^{\prime}} \propto t_{\alpha} t_{\alpha^{\prime}}\right.$ where $t_{\alpha}$ is the hybridization) and only a single channel couples to the dot with $T_{K}=$ $D_{0} \exp \left[\left(3 /\left(2 \rho\left(J_{11}+J_{22}+J_{33}\right)\right)\right]\right.$ (to one-loop order). Since the matrix structure of the general $\mathrm{RG}$ equations respects this property, we can express $g_{\alpha \alpha^{\prime}}(\omega)=3 g_{\omega} n_{\alpha} n_{\alpha^{\prime}}$ where $n_{\alpha}^{2}=J_{\alpha \alpha} /\left(J_{11}+J_{22}+J_{33}\right)$ is the the lead anisotropy parameter (so that $n_{1}^{2}+n_{2}^{2}+n_{3}^{2}=1$ ) and the initial condition is given by $g_{\omega}\left(D_{0}\right)=1 /\left[2 \ln \left(D_{0} / T_{K}\right)\right]$.

For concreteness, let us assume that $\mu_{31}>\mu_{32}>\mu_{21}$ with $\mu_{\alpha \beta} \equiv\left|\mu_{\alpha}-\mu_{\beta}\right|$. When solving the RG equations for $g_{\alpha \beta}\left(\mu_{\beta}\right)$ as a function of the running cutoff $D$, four different regimes have to be considered. First, for $D>\mu_{31}$ one recovers the usual equilibrium RG equations which are easily solved analytically. This is not possible in the second regime, $\mu_{31}>D>\mu_{32}$. However, as $\ln \left[\mu_{31} / \mu_{32}\right] \leq \ln 2 \ll \ln \mu_{31} / T_{K}$ (as $\mu_{32}>\mu_{21}$ by definition), the contributions from this intermediate regime are small and formally subleading in $1 / \ln \left[\mu_{31} / T_{K}\right]$. We can therefore safely take our formulas for $D>\mu_{31}$ and use them even for $D>\mu_{32}$. In the two remaining regimes, $\mu_{32} \geq D \geq \mu_{21}$ and finally $\mu_{21} \geq D \geq 0$ again an analytical solution can be obtained. When calculating $g_{\alpha \beta}(\omega)$ in a second step by simple integration of the RHS of Eq. (2), we do not use any further approximation and treat all four regimes equally (thereby improving also the results for $g_{\alpha \beta}\left(\mu_{\beta}\right)$ for $\mu_{31}>D>\mu_{32}$ as discussed below). The approximation improves with decreasing $\mu_{21}$ and for $\mu_{21}=0$ one recovers correctly the two-lead case for which the one loop RG equations can be solved analytically.

The virtue of having an analytical solution is that it provides insight into how the four independent dimensionless parameters (two $\mu_{\alpha \beta} / T_{K}$ and two $n_{\alpha}^{2}$ ) enter the renormalized $g_{\omega}$ at various stages of the scaling procedure and for what values the strong coupling regime is approached. The role of energy scales is exemplified by the sheer number of regions (already seven for $\mu_{1}<\omega<\mu_{3}$ ) into which the entire frequency range gets divided, most having a different closed form solution for $g_{\omega}$. For the frequency range $\mu_{3} \geq \omega \geq \mu_{2}+\mu_{21}$, we obtain for $D \rightarrow 0$

$$
\begin{aligned}
g_{\omega} & =\frac{n_{1}^{2}}{2 \ln \left[\frac{\mid \widetilde{\omega-\mu_{1} \mid}}{T_{K}}\right]}-\left[n_{1}^{2}+\frac{n_{2}^{2}}{n_{1}^{2}+n_{2}^{2}}\right] \frac{1}{2 \ln \left[\widetilde{\left.\frac{\mu_{32}}{T_{K}}\right]}\right.} \\
& +\frac{n_{2}^{2}}{n_{1}^{2}+n_{2}^{2}} \frac{1}{2\left(n_{1}^{2}+n_{2}^{2}\right) \ln \left[\frac{\left|\widetilde{\omega-\mu_{2}}\right|}{T_{K}}\right]+2 n_{3}^{2} \ln \left[\widetilde{\frac{\mu_{32}}{T_{K}}}\right]} \\
& +\frac{1}{2 n_{3}^{2} \ln \left[\frac{\mid \widetilde{\omega-\mu_{3} \mid}}{T_{K}}\right]+2\left(n_{1}^{2}+n_{2}^{2}\right) \ln \left[\widetilde{\frac{\mu_{32}}{T_{K}}}\right]}
\end{aligned}
$$

while for $\mu_{2} \geq \omega \geq \mu_{1}$ we have

$$
\begin{aligned}
g_{\omega} & =\frac{1}{2 n_{1}^{2} \ln \left[\frac{\mid \widetilde{\omega-\mu_{1} \mid}}{T_{K}}\right]+2 n_{2}^{2} \ln \left[\frac{\widetilde{\mu_{21}}}{T_{K}}\right]+2 n_{3}^{2} \ln \left[\frac{\widetilde{\mu_{32}}}{T_{K}}\right]} \\
& +\frac{1}{2 n_{2}^{2} \ln \left[\frac{\left|\widetilde{\omega-\mu_{2}}\right|}{T_{K}}\right]+2 n_{1}^{2} \ln \left[\widetilde{\frac{\mu_{21}}{T_{K}}}\right]+2 n_{3}^{2} \ln \left[\widetilde{\frac{\mu_{32}}{T_{K}}}\right]} \\
& +\frac{n_{3}^{2}}{2 \ln \left[\frac{\left|\widetilde{\omega-\mu_{3}}\right|}{T_{K}}\right]}-\frac{n_{3}^{2}}{2 \ln \left[\widetilde{\frac{\mu_{32}}{T_{K}}}\right]} \\
& -\frac{1}{2\left(n_{1}^{2}+n_{2}^{2}\right) \ln \left[\frac{\widetilde{\mu_{21}}}{T_{K}}\right]+2 n_{3}^{2} \ln \left[\frac{\widetilde{\mu_{32}}}{T_{K}}\right]} .
\end{aligned}
$$

As a check for our approximative treatment of the $R G$ equations, we compare e.g. $g_{\mu_{3}}$ evaluated by taking $\omega=\mu_{3}$ in Eq. (4), with its initial evaluation described above (not shown). The difference $n_{1}^{2}\left(1 / \ln \left[\widetilde{\mu_{32}} / T_{K}\right]-\right.$ $\left.1 / \ln \left[\widetilde{\mu_{31}} / T_{K}\right]\right) / 2$ is even for the worst case $\left(\mu_{32}=\mu_{31} / 2\right)$ only of order $\ln 2 / \ln ^{2}\left[\mu_{31} / T_{K}\right]$ as expected. As Fig. 1 shows, there is only a very slight overestimation of $g_{\omega}$ as compared to the straightforward numerical solution of Eq. (2) in this case. In all other cases the numerical and analytical curves coincide.

It is important to check under what conditions the problem enters the strong coupling regime where our approach is not valid any more. We can investigate this question analytically by determining the scale $T_{*}^{\alpha}$ at which $g_{\mu_{\alpha}}$ diverges in the absence of $\Gamma$. Analyzing Eqs. (4), (15) , for arbitrary $\mu_{\alpha \beta}>T_{*}$ we obtain

$$
T_{*}=\max _{\alpha=1,2,3}\left[T_{*}^{\alpha}\right] \quad \text { with } \quad \frac{T_{*}^{\alpha}}{T_{K}}=\prod_{\beta \neq \alpha}\left[\frac{T_{K}}{\mu_{\beta \alpha}}\right]^{\left(\frac{n_{\beta}}{n_{\alpha}}\right)^{2}}
$$

while if two of the three leads have the same electrochemical potential (more precisely $\mu_{12}<T_{*}$, say) then

$$
T_{*}=\left[\frac{T_{K}}{\mu_{32}}\right]^{\min \left[\left(n_{2}^{2}+n_{1}^{2}\right) / n_{3}^{2}, n_{3}^{2} /\left(n_{1}^{2}+n_{2}^{2}\right)\right]} .
$$




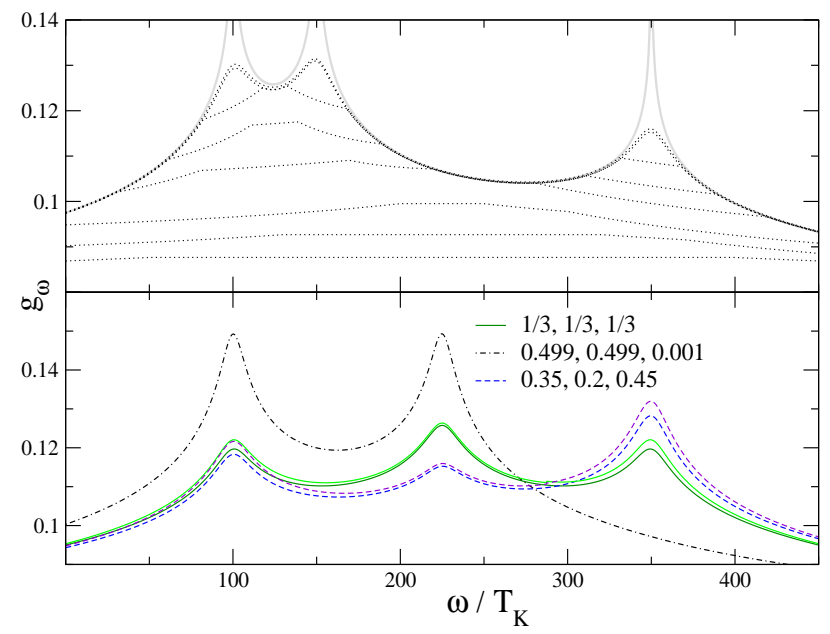

FIG. 1: Upper panel shows the scaling of $g_{\omega}$ as the cut-off is decreased down to zero from $D=300 T_{K}$, for $\mu_{1}=100 T_{K}$, $\mu_{2}=150 T_{K}, \mu_{3}=350 T_{K}$ and $n_{1}=n_{2}=n_{3}$. The light grey plot shows the $g_{\omega}$ obtained if $\Gamma$ were to be $T_{*}$. Lower panel shows the renormalized $g_{\omega}(D=0)$ for $\mu_{1}=100 T_{K}$, $\mu_{2}=225 T_{K}, \mu_{3}=350 T_{K}$ for different $n_{1}, n_{2}, n_{3}$ shown in the legends. In the same line style as numerical plots, also shown in both panels are the plots for $g_{\omega}$ obtained analytically (upper curves when they do not coincide).

For $\Gamma=T_{*}$ at least one of the coupling constants diverges $\propto 1 / \ln \left[\Gamma / T_{*}\right]$ (this is how we determine $T_{*}^{\alpha}$; note that $\widetilde{x} \equiv \sqrt{x^{2}+\Gamma^{2}}$ in the terms $\left.\ln \left[\widetilde{x} / T_{K}\right]\right)$. The perturbative RG is therefore controlled by the ratio $\Gamma / T_{*}$. The larger is the ratio as compared to one, the further away we are from the strong coupling coupling regime, and the more accurate is our analysis.

The upper panel of Fig. 1 is a visual display of the scaling process: $g_{\omega}$ for a generic case is plotted as the cut-off $D$ is gradually decreased; one can see the role of energy scales $\mu_{21}, \mu_{31}$ and $D$, all measured in units of $T_{K}$. To clarify the role of $\Gamma$, we also plot (in light grey) the $g_{\omega}$ obtained if $\Gamma$ were to be $T_{*}$. In the lower panel we illustrate how the interplay between lead anisotropies and potential differences determines the renormalization of $g_{\omega}$. For example, when one lead $\alpha$ is very weakly coupled, there is no feature at $\omega=\mu_{a}$.

The physical quantity of interest is the non-equilibrium conductance matrix, which, within the precision of our approach, can be evaluated by plugging the renormalized coupling constants in the second order perturbative formula to obtain

$$
\begin{aligned}
G_{\alpha \beta} & \equiv e \partial I_{\alpha} / \partial \mu_{\beta}=\frac{\pi^{2} G^{0}}{3} \sum_{\alpha^{\prime} \neq \alpha}\left[\left(\delta_{\alpha \beta}-\delta_{\alpha^{\prime} \beta}\right) g_{\alpha \alpha^{\prime}}^{2}\left(\mu_{\beta}\right)\right. \\
& \left.+\int_{\mu_{\alpha^{\prime}}}^{\mu_{\alpha}} d \varepsilon \frac{\partial}{\partial \mu_{\beta}} g_{\alpha \alpha^{\prime}}^{2}(\varepsilon)\right]
\end{aligned}
$$

where we have restricted to zero temperature and introduced the quantum of conductance, $G^{0}=e^{2} / \pi \hbar$. Let

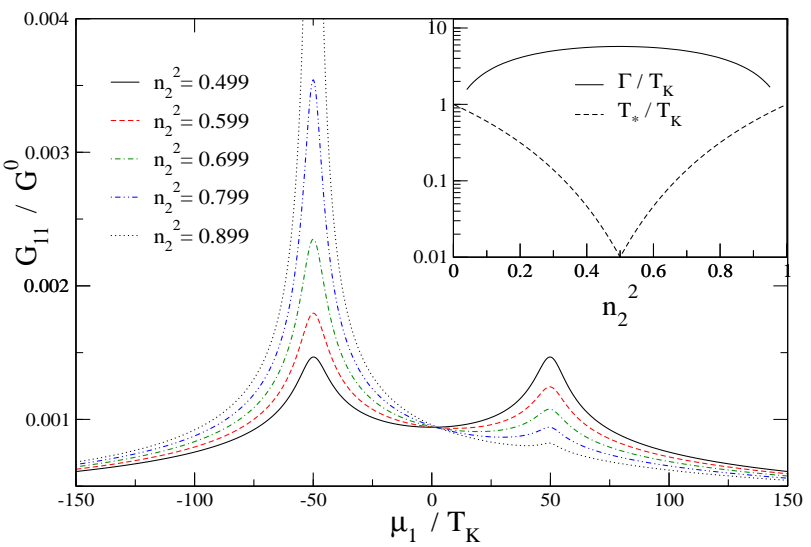

FIG. 2: Conductance $G_{11}$ in the "two-lead spectroscopy" case $\left(n_{1}^{2}=0.002\right)$ for $\mu_{32}=100 T_{K}$ and different values of $n_{2}^{2}$ and $n_{3}^{2}$. Inset shows $\Gamma$ and $T_{*}$ on a log scale, as a function of $n_{2}^{2}$.

us first consider the case when lead one is a "probing lead" i.e. it is very weakly coupled to the dot $\left(n_{1}^{2} \ll 1\right)$. Then $G_{11}\left(\mu_{1}\right)$ is simply given by the first term of Eq. (8) (the second term being negligible owing to very weak dependence of $g^{2}(\varepsilon)$ on $\mu_{1}$ ) and traces the two-lead spectral function of the quantum dot; we have explicitly confirmed this equivalence. If we assume an underlying Anderson model as above, we obtain $G_{11}\left(\mu_{1}\right) / G^{0}=3 \pi^{2} n_{1}^{2}\left(1-n_{1}^{2}\right) g_{\mu_{1}}^{2}$ with $g_{\mu_{1}}$ given by Eq. (5). As $\mu_{1}$ is swept, $G_{11}$ peaks up at $\mu_{2}$ and at $\mu_{3}$ with the peak height at $\mu_{2}$ given by

$$
G_{11}\left(\mu_{1}=\mu_{2}\right)=\frac{3 \pi^{2} n_{1}^{2}\left(1-n_{1}^{2}\right) G^{0}}{\left(2\left(1-n_{3}^{2}\right) \ln \left[\frac{\Gamma}{T_{K}}\right]+2 n_{3}^{2} \ln \left[\widetilde{\frac{\mu_{32}}{T_{K}}}\right]\right)^{2}}
$$

while that at $\mu_{3}$ obtained by interchanging $n_{2} \leftrightarrow n_{3}$ in the above expression. For $n_{1}^{2} \ll 1$ the formula for $T_{*}$ (Eq. (6)) reduces to Eq. (77) which now holds not only for $\mu_{1}=\mu_{2}$ but for all values of $\mu_{1}$.

In Fig. 2 we plot the conductance for different values of the relative anisotropy between couplings to leads two and three, for a given value of potential difference $\mu_{32}$, to emphasize the role of anisotropy. The current from lead two to three is largest for $n_{2} \sim n_{3}=0.5$ and accordingly $\Gamma$ (same for all $\mu_{1}$ as $n_{1}^{2} \ll 1$ ) is maximum at this point. On the other hand, $T_{*}$ is minimum at this point and the ratio of $\Gamma / T_{*}$ largest (see inset). In the extreme anisotropy case when only a single lead $\alpha$ is strongly coupled to the dot, one will measure the equilibrium spectral function $\left(g(\omega)\right.$ is strongly renormalized only at that $\left.\mu_{\alpha}\right)$. In Fig. 3 we show how the conductance changes as the potential difference $\mu_{32}$ is increased, for a given relative anisotropy. To clarify how $\Gamma$ determines the rounding of the peaks, the light line shows $G_{11}$ if $\Gamma$ were to be $T_{*}$ (for $\left.\mu_{32}=100 T_{K}\right)$. With increasing $\mu_{32}$, the value of $\Gamma$ gets larger (since the current increases ) and $T_{*}$ gets smaller, 


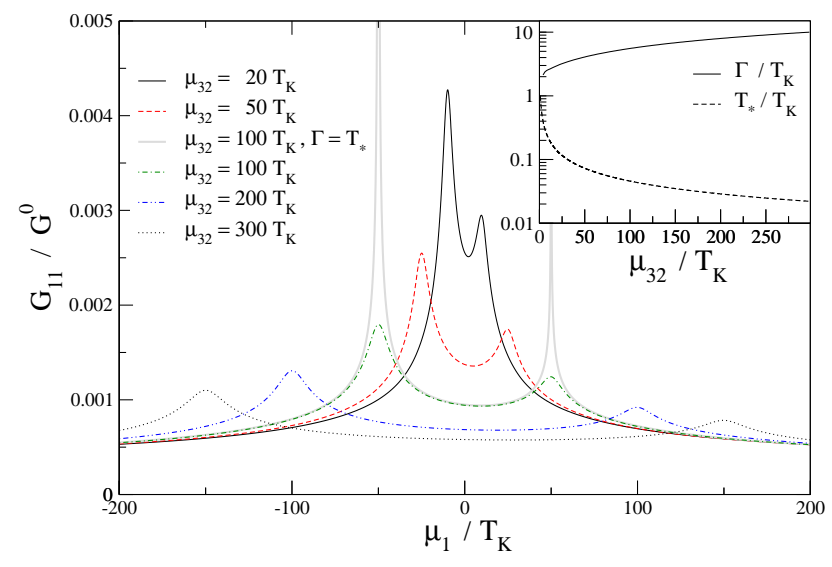

FIG. 3: Conductance $G_{11}$ in the "two-lead spectroscopy" case $\left(n_{1}^{2}=0.002\right)$ for $n_{2}=0.599, n_{3}=0.399$ and different values of $\mu_{32}$. The light curve shows $G_{11}$ if $\Gamma$ were to be $T_{*}$. The inset shows $\Gamma$ and $T_{*}$ on a log scale, as a function $\mu_{32}$.

making the ratio $\Gamma / T_{*}$ increasingly larger (see inset).

To study other cases, we phrase the question: how does the conductance change as we increase the coupling to lead one? We hold the relative anisotropy between leads two and three to be constant as we increase the value of $n_{1}$. As can be seen from Fig. 4 as we start increasing $n_{1}$, the overall conductance $G_{11}$ begins to grow. Further, the second term in Eq. (8) is not negligible as in the "two-lead spectroscopy" case and makes a negative contribution; the inset plots the peak height $G_{11}\left(\mu_{1}=\mu_{3}\right)$ as well as the contribution it gets from the first term to make the increasing deviation between the two evident. As we keep increasing $n_{1}$, we start approaching another limit in which lead one is strongly coupled and leads two and three weakly coupled to the quantum dot: the peaks continue to grow, however, the overall conductance for other values of $\mu_{1}$ begins to drop down. For the sake of presentation we have chosen to focus on $G_{11}$ although it should be clear that analogous arguments hold for the remaining elements of the conductance matrix.

As can be seen from the insets of Figs. 2314 the ratio $\Gamma / T_{*}$ can become quite large for some parameter range making our perturbative RG treatment extremely accurate. Further more, only when the system approaches equilibrium and $T_{*} \rightarrow T_{K}$, do we find that $\Gamma \rightarrow T_{*}$ and the strong coupling regime is approached. This happens either when $\mu_{32} \leq T_{K}$ for the $n_{1}^{2} \ll 1$ case and all $\mu_{\alpha \beta} \leq T_{K}$ for the general case, or in the extreme anisotropy limit when only one of the three leads is strongly coupled to the dot irrespective of the values of $\mu_{\alpha \beta}$. Our finding is in accordance with the expectation for a Kondo model derived from an Anderson model: a strong coupling fixed point exists only in equilibrium [8].

Possible extensions to this work include studying the case of a generalized Kondo model with no underlying Anderson model and considering the effect of excited lev-

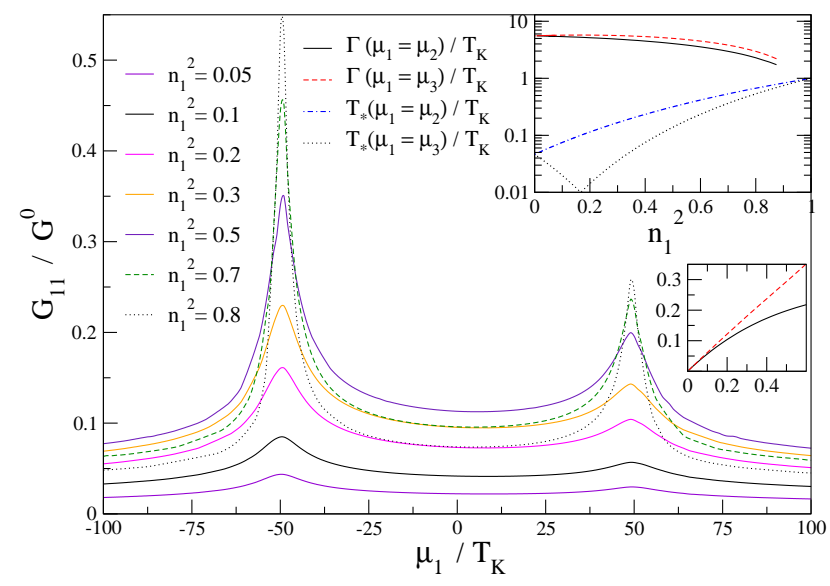

FIG. 4: Plots of $G_{11}$ for increasing values of $n_{1}^{2}$ (ordered by increasing peak heights) for fixed $n_{2}^{2} / n_{3}^{2}=1.5$ and $\mu_{32}=$ $100 T_{K}$. The corner inset shows $\Gamma$ and $T_{*}$ on a log scale, as a function of $n_{1}^{2}$ for $\mu_{1}=\mu_{2}, \mu_{3}$. Other inset shows the peak height $G_{11}\left(\mu_{1}=\mu_{3}\right)$ as well as the part coming only from the first term of Eq. 8] (dashed line) plotted against $n_{1}^{2}$.

els or of a Zeeman field $\left(B \gg T_{K}\right)$. For the specific set-up of a quantum ring geometry enclosing a magnetic flux 14] it might be interesting to investigate the chiral component to the current [20] in the Kondo regime and in non-equilibrium.

We thank K. Ensslin, R. Leturcq, J. Paaske and P. Wölfle for discussions and the DFG for financial support.

[1] S. M. Cronenwett, T. H. Oosterkamp, and L. P. Kouwenhoven, Science 281, 540 (1998).

[2] D. Goldhaber-Gordon et al., Nature 391, 156 (1998).

[3] J. Park et al., Nature 417, 722 (2002).

[4] W. Liang et al., Nature 417, 725 (2002).

[5] N. Schlosser et al., Nature 411, 1024 (2001).

[6] Y. Meir, N. S. Wingreen, and P. A. Lee, Phys. Rev. Lett. 70, 2601 (1993).

[7] A. Kaminski, Y. V. Nazarov, and L. I. Glazman, Phys. Rev. B 62, 8154 (2000).

[8] A. Rosch, J. Kroha, and P. Wölfle, Phys. Rev. Lett. 87, 156802 (2001).

[9] A. Rosch et al., Phys. Rev. Lett. 90, 076804 (2003).

[10] J. Paaske et al., Phys. Rev. B 70, 155301 (2004).

[11] A. Rosch et al., J. Phys. Soc. Jpn. 74, 118 (2005).

[12] Resonant inter-lead processes possible in the presence of a Zeeman field $B \sim V$ do not contribute to the current.

[13] S. De Franceschi et al., Phys. Rev. Lett. 89, 156801 (2002).

[14] R. Leturcq et al., Phys. Rev. Lett. 95, 126603 (2005).

[15] R. C. Toonen et al., 2005, private communication.

[16] Q. F. Sun and H. Guo, Phys. Rev. B 64, 153306 (2001).

[17] E. Lebanon and A. Schiller, Phys. Rev. B 65, 035308 (2002).

[18] S. Y. Cho et al., Phys. Rev. B 68, 125327 (2003).

[19] D. Sanchez and R. López, Phys. Rev. B 71, 035315 (2005).

[20] C. Chamon, M. Oshikawa, and I. Affleck, Phys. Rev. Lett. 91, 206403 (2003). 